\title{
SOLAR NEUTRINOS AND THE JUPITER
}

\author{
W-Y. PAUCHY HWANG \\ The Leung Research Center for Cosmology and Particle Astrophysics, \\ Institute of Astrophysics, Center for Theoretical Sciences, \\ and Department of Physics, National Taiwan University, Taipei 106, Taiwan \\ wyhwang@phys.ntu.edu.tw
}

\begin{abstract}
Judging from the fact that the planet Jupiter is bigger in size than the Earth by $10^{3}$ while is smaller than the Sun by $10^{3}$, the solar neutrinos, when encounter the Jupiter, may have some visible effects. We estimate how much energy/power carried by solar neutrinos get transferred by this unique process. Solar neutrinos, despite of their feeble neutral weak current interactions, might deposit enough energy in the Jupiter.
\end{abstract}

PACS Nos.: 96.40.Tv (Neutrinos and muons), 96.30.Kf (Jupiter), 95.85.Ry (Neutrino, etc.).

\section{Introduction}

Well, these days I only think about simple things, including the research - maybe a person having the experience of cerebral haemorrhage tried to simplify the life in certain way. I should say that on this project I enjoyed very much the collaboration of the visitor Velery Burov during November 2006 to May 2007.

We may look into the eight major planets of our solar system and may wonder where they came from. For instance, the Venus, the Earth, and the Mars look similar - in terms of their densities (the denser planets) and other "observable" properties. On the other hand, the Jupiter and the Saturn might be mini-Suns as can revealed by their densities, because they are quite similar to the Sun. Of course, all the moons should also form or be captured at some time. This kind of "nature" history is worth studying.

Speaking of the Venus, the Earth, and the Mars (the Trio), the most puzzling fact would be why the Venus and the Mars seem to have any life forms of some sort and the Earth is almost ruined by these creatures. In fact, in my classes I usually make jokes that I always wonder that some day monkeys or apes would write down the Einstein equation and solve it.

\section{Solar Neutrinos}

When the Sun is shining on us, a significant fraction of the solar energy get carried away by neutrinos. Solar neutrinos are elusive because they only participate weak 
interactions - so almost all of them pass away by us without being noticed. In fact, solar neutrinos are even more elusive than, e.g., antineutrinos because charged weak interactions operate only selectively between solar neutrinos and the ordinary matter (for example, for going through the reaction $\nu_{e}+(A, Z) \rightarrow(A, Z+1)+e^{-}$ provided that energy-momenta are conserved, provided that the target material has a small amount of initial $(A, Z))$ - they are made of from the matter rather than the antimatter.

For the Jupiter, the composition may be assumed to be sun-like, most of the constituents would be the hydrogen, $H$, and a small fraction the helium, $H e$, or the ionized ones. For the Sun, the mass is $1.9889 \times 10^{30} \mathrm{Kg}$ and the radius $6.961 \times 10^{8} \mathrm{~m}$, while for the Jupiter $1.901 \times 10^{27} \mathrm{Kg}$ and $7.1398 \times 10^{7} \mathrm{~m}$; so the density for the Jupiter is even slightly smaller than the Sun. Thus, the assertion that the Jupiter is a baby Sun is a reasonable assumption.

Let us come to solar neutrinos. Solar neutrinos come from the most important reactions in the so-called $p p$-I chain,

$$
\begin{gathered}
p+p \rightarrow D+e^{+}+\nu_{e}, \quad\left(E_{\nu}^{\max }=0.42 \mathrm{MeV}: \phi_{\nu}=6.0 \times 10^{10} \mathrm{~cm}^{-2} \mathrm{sec}^{-1}\right), \\
p+p+e^{-} \rightarrow D+\nu_{e}, \quad\left(E_{\nu}=1.44: \phi_{\nu}=1.5 \times 10^{8}\right),
\end{gathered}
$$

or from the $p p$-II chain,

$$
{ }^{7} \mathrm{Be}+e^{-} \rightarrow{ }^{7} \mathrm{Li}+\nu_{e}, \quad\left(E_{\nu}=0.86 \mathrm{MeV}: \phi_{\nu}=2.7 \times 10^{9} ; \quad E_{\nu}=0.38: 3.0 \times 10^{8}\right),
$$

or from the $p p$-III chain,

$$
{ }^{8} B \rightarrow{ }^{8} B e *+e^{+}+\nu_{e}, \quad\left(E_{\nu}^{\max }=14.06 ; \phi_{\nu}=3.0 \times 10^{6}\right),
$$

or from the $\mathrm{C}-\mathrm{N}-\mathrm{O}$ cycle,

$$
\begin{array}{ll}
{ }^{13} N \rightarrow{ }^{13} C+e^{+}+\nu_{e}, & \left(E=1.19: 3.0 \times 10^{8}\right), \\
{ }^{15} O \rightarrow{ }^{15} N+e^{+}+\nu_{e}, & \left(E=1.70: 2.0 \times 10^{8}\right) .
\end{array}
$$

Here the neutrino fluxes $\phi_{\nu}$ are measured at the sea level on Earth, in units of $\mathrm{cm}^{-2} \mathrm{sec}^{-1}$. Of course, the electron-like neutrinos may oscillate into muon-like or tao-like specifies but fortunately neutral weak interactions do not differentiate among them; other types of neutrino oscillations, so far less likely, could be relevant though.

The average distance of the planet Jupiter from the Sun is $5.203 a . u$. with the Jupiter year 11.9 our years. The radius of the Jupiter is $71,398 \mathrm{~km}$, much bigger than the Earth's $6,378 \mathrm{~km}$. In terms of the mass, the Jupiter's $1.901 \times 10^{27} \mathrm{Kg}$ is about 300 times heavier than the Earth's $5.974 \times 10^{24} \mathrm{Kg}$. It is believed that the composition of the Jupiter is similar to our Sun, mostly the hydrogen plus a certain fraction of the helium. 
Therefore, when solar neutrinos encounter the Jupiter, we anticipate that the following weak interactions will dominate:

$$
\nu+p \rightarrow \nu+p, \quad \nu+{ }^{4} \mathrm{He} \rightarrow \nu+{ }^{4} \mathrm{He},
$$

while the reaction $\nu+e^{-} \rightarrow \nu+e^{-}$would serve as a small correction.

\section{Estimate on the Mean Free Paths}

For the neutral-current weak reaction induced by solar neutrinos on the protons,

$$
\nu\left(p_{\nu}\right)+p(p) \rightarrow \nu\left(p_{\nu}^{\prime}\right)+p\left(p^{\prime}\right)
$$

the transition amplitude is given by ${ }^{1}$

$$
T=\frac{G}{\sqrt{2}} i \bar{u}_{\nu}\left(p_{\nu}^{\prime}\right) \gamma_{\lambda}\left(1+\gamma_{5}\right) u_{\nu}\left(p_{\nu}\right) \cdot<p\left(p^{\prime}\right)\left|N_{\lambda}\right| p(p)>.
$$

We may proceed to parameterize the neutral-current matrix element as follows: ${ }^{1}$

$$
\begin{gathered}
<p\left(p^{\prime}\right)\left|N_{\lambda}(0)\right| p(p)> \\
=i \bar{u}\left(p^{\prime}\right)\left\{\gamma_{\lambda} f_{V}^{N}\left(q^{2}\right)-\frac{\sigma_{\lambda \eta} q_{\eta}}{2 m_{p}} f_{M}^{N}\left(q^{2}\right)+\gamma_{\lambda} \gamma_{5} f_{A}^{N}\left(q^{2}\right)+\frac{i 2 M q_{\lambda} \gamma_{5}}{m_{\pi}^{2}} f_{P}^{N}\left(q^{2}\right)\right\} u(p),
\end{gathered}
$$

with $q^{2} \equiv \vec{q}^{2}-q_{0}^{2}, q_{\lambda}=\left(p^{\prime}-p\right)_{\lambda}$, and $2 M=m_{p}+m_{n}$. Here $f_{V}^{N}\left(q^{2}\right), f_{M}^{N}\left(q^{2}\right)$, $f_{A}^{N}\left(q^{2}\right)$, and $f_{P}^{N}\left(q^{2}\right)$, respectively, the (neutral-current) vector, weak magnetism, axial, and pseudoscalar form factors. The differential cross section is given by

$$
\begin{gathered}
\frac{d \sigma}{d \Omega_{\nu}}(\nu+p \rightarrow \nu+p) \\
=\frac{G^{2}\left(E_{\nu}^{\prime}\right)^{2}}{2 \pi^{2}} \frac{E_{\nu}^{\prime}}{E_{\nu}}\left\{\left[\left(f_{V}^{N}\left(q^{2}\right)\right)^{2}+\left(f_{M}^{N}\left(q^{2}\right)\right)^{2} \frac{q^{2}}{4 m_{p}^{2}}+\left(f_{A}^{N}\left(q^{2}\right)\right)^{2}\right] \cos ^{2} \frac{\theta_{\nu}}{2}\right. \\
+2\left[\left(f_{V}^{N}\left(q^{2}\right)+f_{M}^{N}\left(q^{2}\right)\right)^{2} \frac{q^{2}}{4 m_{p}^{2}}+\left(f_{A}^{N}\left(q^{2}\right)\right)^{2}\left(1+\frac{q^{2}}{4 m_{p}^{2}}\right)\right. \\
\left.\left.+4 \frac{E_{\nu}^{\prime}}{m_{p}}\left(1+\frac{E_{\nu}}{m_{p}} \sin ^{2} \frac{\theta_{\nu}}{2}\right) f_{A}^{N}\left(q^{2}\right)\left(f_{V}^{N}\left(q^{2}\right)+f_{M}^{N}\left(q^{2}\right)\right)\right] \sin ^{2} \frac{\theta_{\nu}}{2}\right\} .
\end{gathered}
$$

In the tree approximation in the standard model of particle physics, we have

$$
N_{\lambda}=\left(1-2 \sin ^{2} \theta_{W}\right) I_{\lambda}^{3}-\sin ^{2} \theta_{W} Y_{\lambda}+I_{\lambda}^{3(5)}-\frac{1}{2} Y_{\lambda}^{s}-\frac{1}{2} Y_{\lambda}^{s(5)},
$$

so that, for example,

$$
\begin{gathered}
f_{V}^{N}\left(q^{2}\right)=\left(1-2 \sin ^{2} \theta_{W}\right) \cdot \frac{1}{2}\left(e_{p}\left(q^{2}\right)-e_{n}\left(q^{2}\right)\right)-\sin ^{2} \theta_{W} \cdot\left(e_{p}\left(q^{2}\right)+e_{n}\left(q^{2}\right)\right)-\frac{1}{2} f_{V}^{S}\left(q^{2}\right) . \\
f_{M}^{N}\left(q^{2}\right)=\left(1-2 \sin ^{2} \theta_{W}\right) \cdot \frac{1}{2}\left(\mu_{p}\left(q^{2}\right)-\mu_{n}\left(q^{2}\right)\right)-\sin ^{2} \theta_{W} \cdot\left(\mu_{p}\left(q^{2}\right)-\mu_{n}\left(q^{2}\right)\right)-\frac{1}{2} f_{M}^{S}\left(q^{2}\right) . \\
f_{A}^{N}\left(q^{2}\right)=\frac{1}{2} f_{A}\left(q^{2}\right)-\frac{1}{2} f_{A}^{S}\left(q^{2}\right) .
\end{gathered}
$$


As a reasonable estimate, we could use $q^{2} \approx 0$ and neglect all terms higher order in $q^{2} / m_{p}^{2}$ and $E_{\nu} /\left(2 m_{p}\right)$. The integration over $d \Omega$ yields

$$
\begin{aligned}
\sigma \cong & \frac{G^{2} E_{\nu}^{2}}{\pi} \cdot\left\{\left(\bar{f}_{V}^{2}+\bar{f}_{A}^{2}+\ldots\right)\left(1+\frac{2 E_{\nu}}{m_{p}}\right)^{-1}\right. \\
& \left.+\left(2 \bar{f}_{A}^{2}+\ldots\right)\left(1+\frac{2 E_{\nu}}{m_{p}}\right)^{-2}\right\} \\
\approx & 1.686 \times 10^{-20} \cdot\left(\bar{f}_{V}^{2}+3 \bar{f}_{A}^{2}\right) \cdot\left(\frac{E_{\nu}}{1 M e V}\right)^{2} \cdot \text { barn },
\end{aligned}
$$

where $\bar{f}_{V}$ and $\bar{f}_{A}$ are suitable averages of $f_{V}^{N}\left(q^{2}\right)$ and $f_{A}^{N}\left(q^{2}\right)$, respectively.

The neutrinos could come from either the three-body modes (i.e. the $\beta^{+}$decays) or the two-body modes (such as the $\beta^{+}$capture reactions). For the three-body modes, we could use the phase factors to do very good estimates for the neutrino spectra; we adopt this approximation in this paper.

Our estimate, from Eqs. (1)-(6), for the average flux times the cross section, $\phi_{\nu} \sigma$, is given by

$$
\phi_{\nu} \sigma=4.838 \times 10^{-36}\left(\bar{f}_{V}^{2}+3 \bar{f}_{A}^{2}\right) \sec ^{-1} .
$$

The average density of the Jupiter is $1.2469 \mathrm{gm} / \mathrm{cm}^{3}$. The inverse of the mean free path $n \sigma$ is given by

$$
n \sigma=2.102 \times 10^{-36}\left(\bar{f}_{V}^{2}+3 \bar{f}_{A}^{2}\right) c m^{-1} .
$$

The neutrino flux suitably weighted by the energy factor, measured on the surface of the Jupiter, is

$$
\phi_{\nu}=2.869 \times 10^{8} \mathrm{~cm}^{-2} \mathrm{sec}^{-1} .
$$

This factor is already used before, calculated from from Eqs. (1)-(6) adjusted by the distance from the Jupiter and the Sun.

As another estimate, we could compare how much energy the solar neutrinos deposit in the Jupiter to that in the Earth,

$$
\left(\frac{1}{5.203}\right)^{2} \times\left(\frac{71,398 k m}{6,378 k m}\right)^{3}=51.82,
$$

modulated by small difference in the densities.

Another interesting question to ask: The Sun produces a lot neutrinos (antineutrinos) per unit of time but we could ask which stellar object catches the most of them? How many of them get lost in the empty space? Well, the Jupiter does come into play.

\section{Discussions}

Unless the neutrino species would oscillate into the antineutrino species and the Jupiter is a mini-Sun (in terms of the composition, most hydrogens and a little bit of heliums), the relevant weak interactions are relatively simple in our problem - 
only neutral-current weak interactions and, in terms of the energy range, mostly the elastic channels. The simplification also comes from the constitution of the Jupiter - a mini-Sun, consisting mostly of hydrogen and helium.

Of course, whether neutrino-antineutrino oscillation occurs is an important issue. Antineutrinos in this energy range are more active than the corresponding neutrinos. That should belong to a different paper.

Indeed, neutrino oscillations is now established to be of importance in the Sun - thus, we could speculate that it is also true in the Jupiter, the Mini-Sun, a factor of 10 smaller (in diameter). The scenario for the oscillations is still unknown and yet to be established, and we feel that the oscillations into antineutrinos $(\Delta L=2)$ or into sterile species, if happens, would add a lot of fun in the game. In this paper, we have no room to discuss all these questions involved.

\section{Acknowledgments}

The Taiwan CosPA project is funded by the Ministry of Education (89-N-FA01-1-0 up to 89-N-FA01-1-5) and the National Science Council (NSC 96-2752-M-002-007PAE). This research is also supported in part as another National Science Council project (NSC 96-2112-M-002-023-MY3).

\section{References}

1. For notations, see, e.g., W-Y. P. Hwang, Phys. Rev. D38, 3427 (1988); or in the textbook, W-Y. Pauchy Hwang, "Introduction to Theoretical Particle Physics" (World Scientific, Singapore, 2008), in press. 